\title{
Characterization of cerebral hemodynamic phases following severe head trauma: hypoperfusion, hyperemia, and vasospasm
}

Neil A. Martin, M.D., Ravish V. Patwardhan, M.D., Michael J. Alexander, M.D., Cynthia Zane Africk, M.D., Jae Hong Lee, M.D., Ehud Shalmon, M.D., David A. Hovda, Ph.D., and Donald P. Becker, M.D.

University of California at Los Angeles Cerebral Blood Flow Laboratory, Brain Injury Research Center, and Division of Neurosurgery, University of California at Los Angeles School of Medicine, Los Angeles, California

The extent and timing of posttraumatic cerebral hemodynamic disturbances have significant implications for the monitoring and treatment of patients with head injury. This prospective study of cerebral blood flow (CBF) (measured using ${ }^{133}$ Xe clearance) and transcranial Doppler (TCD) measurements in 125 patients with severe head trauma has defined three distinct hemodynamic phases during the first 2 weeks after injury. The phases are further characterized by measurements of cerebral arteriovenous oxygen difference $\left(\mathrm{AVDO}_{2}\right)$ and cerebral metabolic rate of oxygen $\left(\mathrm{CMRO}_{2}\right)$. Phase I (hypoperfusion phase) occurs on the day of injury (Day 0 ) and is defined by a low $\mathrm{CBF}_{15}$ calculated from cerebral clearance curves integrated to 15 minutes (mean $\mathrm{CBF}_{15} 32.3 \pm 2 \mathrm{ml} / 100 \mathrm{~g} /$ minute), normal middle cerebral artery (MCA) velocity (mean $\mathrm{V}_{\text {MCA }} 56.7 \pm 2.9 \mathrm{~cm} /$ second), normal hemispheric index (mean HI $1.67 \pm 0.11$ ), and normal $\mathrm{AVDO}_{2}\left(\right.$ mean $\left.\mathrm{AVDO}_{2} 5.4 \pm 0.5 \mathrm{vol} \%\right)$. The $\mathrm{CMRO}_{2}$ is approximately $50 \%$ of normal (mean $\mathrm{CMRO}_{2} 1.77 \pm 0.18 \mathrm{ml} / 100 \mathrm{~g} /$ minute) during this phase and remains depressed during the second and third phases. In Phase II (hyperemia phase, Days 1-3), CBF increases (46.8 $\pm 3 \mathrm{ml} / 100 \mathrm{~g} / \mathrm{minute}$ ), $\mathrm{AVDO}_{2}$ falls $(3.8 \pm 0.1 \mathrm{vol} \%), \mathrm{V}_{\mathrm{MCA}}$ velocity rises $(86 \pm 3.7 \mathrm{~cm} / \mathrm{second})$, and the HI remains less than 3 $(2.41 \pm 0.1)$. In Phase III (vasospasm phase, Days 4-15), there is a fall in CBF $(35.7 \pm 3.8 \mathrm{ml} / 100$ $\mathrm{g} /$ minute), a further increase in $\mathrm{V}_{\mathrm{MCA}}(96.7 \pm 6.3 \mathrm{~cm} / \mathrm{second})$, and a pronounced rise in the $\mathrm{HI}(2.87 \pm$ $0.22)$.

This is the first study in which CBF, metabolic, and TCD measurements are combined to define the characteristics and time courses of, and to suggest etiological factors for, the distinct cerebral hemodynamic phases that occur after severe craniocerebral trauma. This research is consistent with and builds on the findings of previous investigations and may provide a useful temporal framework for the organization of existing knowledge regarding posttraumatic cerebrovascular and metabolic pathophysiology.

Key Words * cerebral blood flow * cerebral metabolism * head injury * hyperemia * ischemia * transcranial Doppler ultrasound * vasospasm 
Disturbances of the cerebral circulation play a key role in the pathophysiology of head injury. Ischemic brain damage has been identified histologically in approximately $90 \%$ of patients who die following closed head injury.[21] Ischemia has been called the single most important secondary insult, and several clinical head trauma studies have linked low cerebral blood flow (CBF) to poor

outcome. $[8-10,26,42,46,47,49]$ Several factors may result in posttraumatic cerebral ischemia: increased intracranial pressure (ICP), systemic arterial hypotension, cerebral edema, focal tissue compression from hematomas, and microvascular pathology.[19,23,35,39,48,53] Another potential cause of cerebral ischemia is the posttraumatic spasm of the large cerebral arteries (vasospasm), which has been documented by angiographic and transcranial Doppler (TCD) studies.[33,36,37,57,60,63] Posttraumatic vasospasm has been associated with the finding of cerebral infarction on late computerized tomography (CT) scans and with poor outcome in some head-injured patients.[12,36,37,50] Cerebral hyperemia has also been observed following head injury and has been related to cerebral swelling, increased ICP, and poor outcome.[11,17,29,39,43] For several reasons it is critically important to understand the evolution and timing of these trauma-induced physiological derangements. First, the temporal correlation of cerebral circulatory changes with other physiological and clinical trends will provide new insights into the pathophysiology of brain injury. Second, clinical monitoring techniques that focus on ischemia, hyperemia, or vasospasm must be planned with the proper time courses in mind. Third, therapeutic strategies intended to counteract specific circulatory disturbances must be administered at the appropriate time.

Although previously published studies have defined certain elements of the cerebral hemodynamic response to head injury, none has provided a comprehensive, day-to-day description of trauma-induced cerebral circulatory disturbances. The first study that combined an analysis of CBF and TCD measurements to delineate the time-dependent posttraumatic cerebral hemodynamic changes that occur after craniocerebral trauma was presented by this research group in 1992.[69] That pilot project defined three distinct phases: I, hypoperfusion during the first 24 hours (postinjury Day 0); II, hyperemia on postinjury Days 1 to 3; and III, vasospasm during Days 4 to 14. To validate these preliminary findings, the University of California at Los Angeles (UCLA) Brain Injury Research Center initiated a prospective study of trauma-induced cerebral circulatory disturbances in August 1992. We have analyzed the physiological measurements obtained in 125 severely head injured patients and have confirmed the existence of these three distinct hemodynamic phases. These results indicate that there is a stereotypical pattern of cerebral hemodynamic alterations that is generally found after severe traumatic brain injury. Recognition of this phasic pattern should prove useful in the future for the planning of monitoring in patients suffering from head trauma and for the design of time-dependent therapeutic strategies.

\section{CLINICAL MATERIAL AND METHODS}

\section{Study Design and Patient Population}

The data presented in this report were collected prospectively during the evaluation and management of 125 patients with severe closed head injury who were admitted to UCLA or Harbor/UCLA Medical Centers between August 1992 and January 1995. This study included two subgroups. The first group included 91 patients who underwent serial CBF, cerebral metabolism, and/or TCD evaluations as part of their enrollment (with family consent) in an institutional review board-approved prospective traumatic brain injury cohort study of epidemiological, clinical, and physiological predictors of long-term neuropsychological outcome (the UCLA Brain Injury Research Center [BIRC] project). The second group was composed of 34 patients who underwent CBF, cerebral metabolic, and/or TCD evaluations as 
part of their clinical management. Because the clinical characteristics and the temporal pattern defined by the CBF and TCD studies in these two groups were similar, the groups were combined for the analyses reported in this paper. The mean age of the patients in this study was $33 \pm 14.6$ years (range 16-80 years), and $83 \%$ of the patients were male. Admission Glasgow Coma Scale[58] (GCS) scores ranged from 3 to 8 (median 6).[35] Patients with multisystem trauma were not excluded. Twelve patients in whom CBF and TCD studies were obtained died during the course of their hospitalization. All studies, including those obtained as patients neared brain death, were included in this analysis.

\section{Management Protocol}

The patients were admitted directly to the neurosurgical intensive care unit (ICU), after an initial head CT scan was obtained, or postoperatively. Craniotomies were performed for removal of hematomas and/or other decompressive procedures in 60 patients. Intracranial pressure was measured in 106 patients by means of a ventriculostomy (Becker EDMS II drainage system; Pudenz-Schulte Medical, Goleta, CA) or a fiberoptic ICP monitor (model V240; Camino Laboratories, San Diego, CA). The therapeutic protocol in the ICU included maintenance of ICP below $20 \mathrm{~mm} \mathrm{Hg}$ and cerebral perfusion pressure (CPP) above $70 \mathrm{~mm} \mathrm{Hg}$. Intracranial hypertension (ICP > $20 \mathrm{~mm} \mathrm{Hg}$ ) was treated in a sequential fashion: positioning the patient with the head elevated to $30^{\circ}$ and inducing mild hyperventilation $\left(\mathrm{PaCO}_{2} 30-35\right.$ $\mathrm{mm} \mathrm{Hg}$ ), followed by ventricular CSF drainage, narcotic sedation, neuromuscular blockade, and bolus mannitol therapy as needed. The CPP was kept above $70 \mathrm{~mm} \mathrm{Hg}$ by controlling the ICP and with the use of intravascular volume expansion and vasopressor therapy when necessary. In three of the study patients with intractably increased ICP (ICP > $30 \mathrm{~mm} \mathrm{Hg}$ for 30 minutes), barbiturate coma (titrated to electroencephalogram burst suppression) was induced at some point during the hospital stay.

The management protocol at UCLA and Harbor/UCLA Medical Centers includes intensive cerebral hemodynamic monitoring. Ideally, CBF and TCD studies were performed as soon as possible after admission and repeated regularly until the patient died, recovered, or was either transferred or discharged. Recently, jugular bulb venous blood sampling and calculation of brain arteriovenous oxygen difference $\left(\mathrm{AVDO}_{2}\right)$ have become a standard component of intensive monitoring to assess the adequacy of cerebral oxygen delivery. Significant abnormalities in CBF, TCD results, or cerebral $\mathrm{AVDO}_{2}$ measurements prompted a careful assessment of the relevant physiological parameters (arterial blood pressure, ICP, $\mathrm{PaCO}_{2}, \mathrm{PaO}_{2}$ and oxygen saturation, and hematocrit). These parameters were optimized when the $\mathrm{CBF}$ and TCD measurements demonstrated a marked disturbance in the cerebral hemodynamic status (for example, hyperventilation was reduced and low $\mathrm{PaCO}_{2}$ was corrected in patients with markedly reduced CBF). Beyond this, no specific treatment was administered for TCD-diagnosed vasospasm.

\section{Measurement Techniques}

Transcranial Doppler Ultrasound. The middle cerebral artery (MCA) and extracranial (EC) internal carotid artery (ICA) were insonated using a 2-MHz pulsed TCD device (Cerebrovascular Diagnostic System; Neurogard/Nicolet, Madison, WI) via transtemporal and submandibular methods respectively, as previously described by Aaslid, et al.[1] The flow parameters that were recorded and analyzed included the mean velocities (the time mean of the peak velocities over the course of four cardiac cycles) for the $\mathrm{MCA}\left(\mathrm{V}_{\mathrm{MCA}}\right)$ and extracranial ICA $\left(\mathrm{V}_{\mathrm{EC}-\mathrm{ICA}}\right)$. The pulsatility index $(\mathrm{PI}=[$ systolic velocity - diastolic velocity]/mean velocity) was also measured for each of these vessels; normal MCA values are $0.71 \pm$ 
0.13, whereas extracranial ICA values are $0.74 \pm 0.13$.[56] A direct relationship between the PI and increased peripheral vascular resistance has been demonstrated, if other hemodynamic factors, such as arterial pulse pressure, remain constant.[30]

Because the extracranial ICA is not involved in vasospasm, velocity changes in this vessel result from changes in $\mathrm{CBF}$ rather than from changes in arterial diameter. Therefore $\mathrm{V}_{\mathrm{EC}-\mathrm{ICA}}$ can be used as an index of $\mathrm{CBF}$ and the ratio of $\mathrm{V}_{\mathrm{MCA}}$ to $\mathrm{V}_{\mathrm{EC}-\mathrm{ICA}}\left(\mathrm{V}_{\mathrm{MCA}} / \mathrm{V}_{\mathrm{EC}-\mathrm{ICA}}\right)$ can be used to distinguish hyperemia from vasospasm. In hyperemia, both $\mathrm{V}_{\mathrm{MCA}}$ and $\mathrm{V}_{\mathrm{EC} \text {-ICA }}$ are increased, and the ratio remains normal $(1.7 \pm$ 0.4).[31] In vasospasm, the $\mathrm{V}_{\mathrm{MCA}}$ is increased, the $\mathrm{V}_{\mathrm{EC} \text {-ICA }}$ is unchanged or decreased, and thus the ratio is increased. Lindegaard, et al.,[31] have written that a $\mathrm{V}_{\mathrm{MCA}} / \mathrm{V}_{\mathrm{EC}-\mathrm{ICA}}$ ratio greater than 3 corresponds to angiographically visible spasm. This ratio has been referred to as the "Lindegaard ratio" or the "hemispheric index" (HI). In this report the presence of vasospasm is determined by the simultaneous finding of $\mathrm{V}_{\mathrm{MCA}}$ greater than $120 \mathrm{~cm} / \mathrm{second}$, and $\mathrm{V}_{\mathrm{MCA}} / \mathrm{V}_{\mathrm{EC}-\mathrm{ICA}}$ greater than 3 .

As a part of the routine clinical assessment, TCD studies were generally performed within 24 to 48 hours of admission and serial studies were performed for up to 3 weeks or more after admission. Patients enrolled in the BIRC project were studied, as much as possible, according to a prospectively established schedule (on Days $0,1,2,3,5,7,9,10,12$, and 14). A total of 789 TCD studies were performed in 120 patients.

Global CBF Measurements. Intravenous ${ }^{133} \mathrm{Xe}-\mathrm{CBF}$ studies were performed using a portable apparatus (Cortexplorer 10; Ceretronix, Randers, Denmark) as previously described.[3,9,26,42-44] Approximately 20 to $30 \mathrm{mCi}$ of ${ }^{133} \mathrm{Xe}$ gas dissolved in saline was injected intravenously for each study. The portable CBF unit was used to estimate cerebral perfusion by analyzing ${ }^{133} \mathrm{Xe}$ wash-out curves measured over 11 minutes by five probes positioned over each cerebral hemisphere (primarily over the MCA territory). Analytic computer software was then used to extrapolate the data to 15 minutes, referencing the brain clearance curves to the clearance curve of exhaled end-tidal xenon-133 (which is used to estimate the arterial concentration of xenon-133). A modified height-over-area method was used to calculate $\mathrm{CBF}_{15}$. This CBF index represents the mean flow of both fast- and slow-clearing compartments, is insensitive to "slippage," is more stable than individual compartment parameters in pathological conditions, correlates well with CBF measurements made using stable Xe-CT scanning, and has been used extensively in head trauma research.[3,8,9,26,42-44] Blood pressure, hematocrit, ICP, and $\mathrm{PaCO}_{2}$ were recorded at the time of each study. The $\mathrm{CBF}_{15}$ values for all 10 detectors were averaged and the mean was judged to represent the global $\mathrm{CBF}$ for that test. The $\mathrm{CBF}_{15}$ values were corrected to a standard $\mathrm{PaCO}_{2}$ of $34 \mathrm{~mm} \mathrm{Hg}$ ("corrected $\mathrm{CBF}_{15}$ "), assuming a $3 \%$ increase in $\mathrm{CBF}$ for each 1-mm $\mathrm{Hg}$ increase in $\mathrm{PaCO}_{2}$ (following the convention of Obrist and colleagues[43]).

A total of $313 \mathrm{CBF}$ studies were performed in 69 of the 125 patients. Patients were missed during periods when a trained technologist was not available or when equipment malfunction precluded study, indicating that these patients were missed at random. The subgroup in which CBF studies were performed is representative of the group as a whole because the patients did not differ significantly in age (33.3 \pm 15.8 years), gender ( $83 \%$ male), or median admission GCS score (6) from the entire study cohort or from the patients who underwent TCD testing.

The first study on a given head-injured patient was typically performed within 48 hours of admission and 
many were performed within 24 hours of admission (with three studies within 12 hours). For clinical assessment, serial studies were performed in patients over the subsequent weeks. Patients in the BIRC project were studied, as much as possible, according to a prospectively established schedule (on Days 0 , $1,3,5,10$, and 14). Scheduled studies were missed or rescheduled for a variety of reasons such as delays in obtaining consent, patient unavailability during scheduled surgery, equipment malfunction, and radioisotope unavailability. Many CBF (and TCD) studies scheduled for the 2nd week after injury were not performed because stable patients were transferred to other medical or rehabilitation facilities (often at the request of managed health care organizations)--this is a source of bias that must be recognized.

Jugular Bulb Catheter Studies. Jugular bulb catheterization was included as a part of routine management and the BIRC investigative protocol relatively recently. As soon as possible after admission to the ICU, retrograde catheterization of the internal jugular vein was accomplished using a No. 4 French fiberoptic intravascular oxygen saturation catheter (model U440; Abbott Laboratories, No. Chicago, IL). The catheter was introduced percutaneously and advanced to the jugular bulb at the base of the skull. The position was verified by lateral skull x-ray film. While the catheter was in place (usually for 5-7 days after insertion, occasionally up to postinjury Days 10-12), jugular venous and radial arterial blood samples were drawn every 8 hours, and values for $\mathrm{AVDO}_{2}$ were calculated.[26,27] Simultaneous $\mathrm{PaCO}_{2}$ readings were recorded. The $\mathrm{AVDO}_{2}$ values were corrected to a $\mathrm{PaCO}_{2}$ of $34 \mathrm{~mm} \mathrm{Hg}$, assuming an increase of $3 \%$ in $\mathrm{AVDO}_{2}$ for every 1-mm $\mathrm{Hg}$ decrease in $\mathrm{PaCO}_{2}$ (again after the convention of Obrist and colleagues[43]). The $\mathrm{CMRO}_{2}$ was estimated from the product of $\mathrm{AVDO}_{2}$ and the global value for $\mathrm{CBF}_{15} \cdot[43]$ 

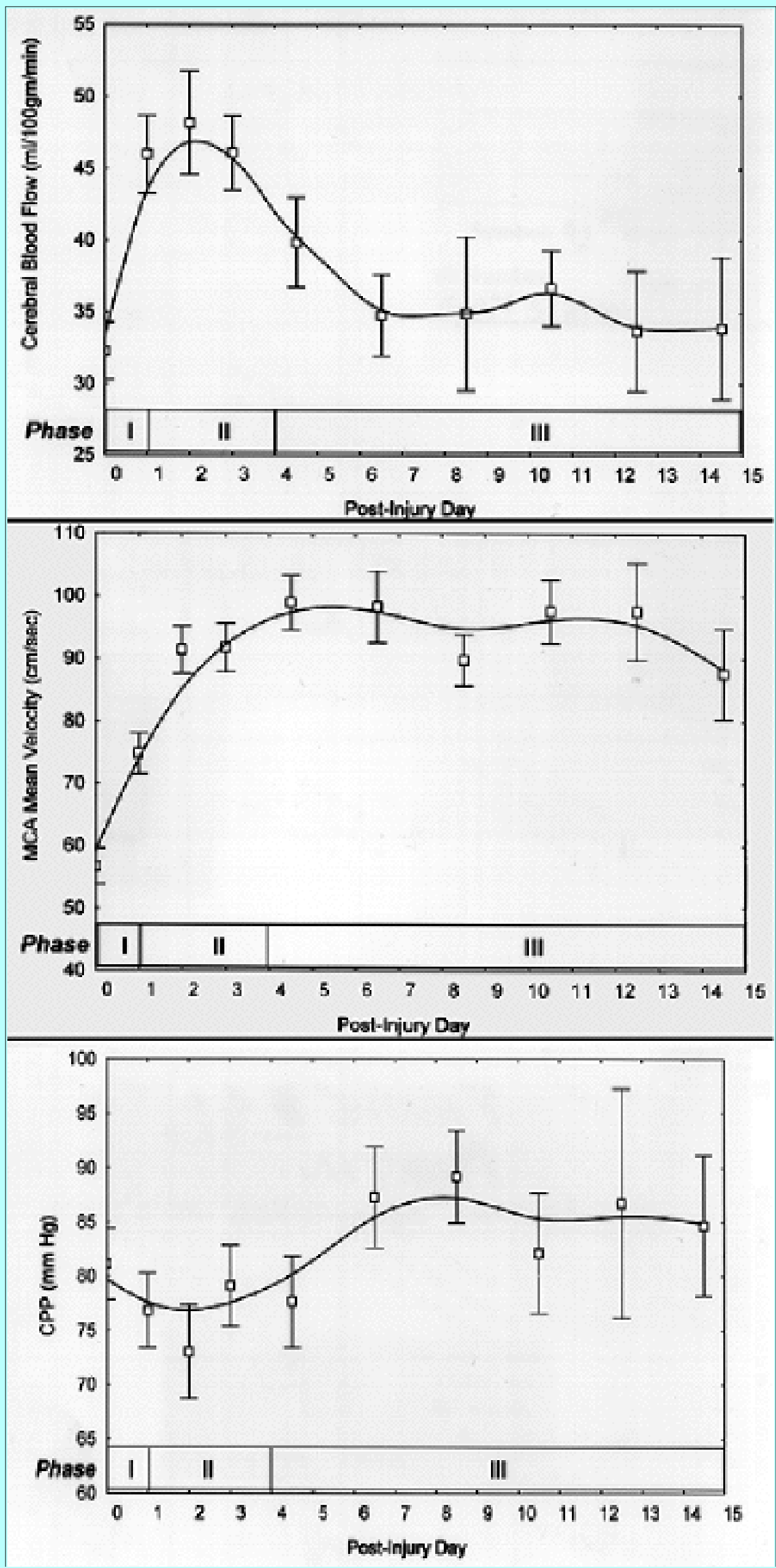
Fig. 1. Graphs displaying the time courses of posttraumatic $\mathrm{CBF}$ (adjusted to a $\mathrm{PaCO}_{2}$ of 34 $\mathrm{mm} \mathrm{Hg}$ ), MCA velocity, and CPP observed after severe head trauma. Upper: The mean CBF values $( \pm$ SEM $)$ for all patients who were studied each day are represented. Center: The mean MCA velocity values ( \pm SEM) were measured using TCD ultrasonography. Lower: The daily mean CPP values were arrived at using the following formula: $\mathrm{CPP}=$ mean arterial pressure - mean ICP $( \pm$ SEM). Postinjury Day 0 designates the first 24 hours after injury. The three cerebral hemodynamic phases are indicated at the bottom of the graph (Phase I, hypoperfusion; Phase II, hyperemia; and Phase III, vasospasm).

A total of 369 measurements of $\mathrm{AVDO}_{2}$ were made in 26 patients. This subgroup appears to be representative of the group as a whole (mean age 36.6 \pm 17.7 years; median GCS score $5 ; 77 \%$ male).

Measurements of ICP and CPP. Measurements of ICP were acquired during the time of CBF study. The $\mathrm{CPP}$ values were calculated from the ICP and the mean arterial pressure measurements recorded at the time of study.

\section{Definition of Timing of Posttraumatic Cerebral Hemodynamic Phases}

The daily values for $\mathrm{CBF}, \mathrm{TCD}$, and $\mathrm{AVDO}_{2}$ measurements and physiological parameters were grouped into the three temporal phases as they were defined by our pilot project.[69] That preliminary study, based on CBF and TCD studies in 32 patients suffering from head trauma, identified three posttraumatic cerebral hemodynamic phases: Phase I, first 24 hours after injury (postinjury Day 0); Phase II, 24 to 96 hours after injury (postinjury Days 1-3); and Phase III (postinjury Days 4-14). The pilot study also included a fourth phase (postinjury Weeks 2 and 3) during which the CBF and TCD abnormalities began to resolve. Because of the paucity and irregular timing of CBF and TCD studies beyond 14 days postinjury, we have not included these late measurements in this report.

\section{Statistical Analysis}

The values for CBF, TCD, metabolic, and physiological measurements were sorted by postinjury day and the daily mean values ( \pm standard error of the mean [SEM]) were calculated. Because fewer daily measurements were obtained after postinjury Day 3, from that point onward the measurements for 2 consecutive days were pooled and the mean values $( \pm$ SEM) for that 2-day period were calculated. The daily mean values ( \pm SEM) were used to generate the time-course graphs (Fig. 1). The curves were generated using a least-squares polynomial curve-fitting function. The values for the various parameters were also grouped according to phase, and the mean values ( \pm SEM) for each phase were calculated (Tables 1 and 2).

To describe the distribution of CBF and TCD measurements within the three phases, all studies performed during each phase were sorted into quantitatively stratified groups defined by the values indicated in Fig. 2. The relative frequency of studies in each group, for each phase, are illustrated in this figure.

Two-sample, equal variance-based t-tests with Bonferroni correction were used to confirm that the three hemodynamic phases, based on $\mathrm{CO}_{2}$-corrected $\mathrm{CBF}_{15}, \mathrm{~V}_{\mathrm{MCA}}$, and $\mathrm{HI}$, were separate and distinct. These tests were also used to assess any statistically significant differences in $\mathrm{AVDO}_{2}, \mathrm{CMRO}_{2}, \mathrm{PI}$, ICP, and $\mathrm{CPP}$ values between phases. 

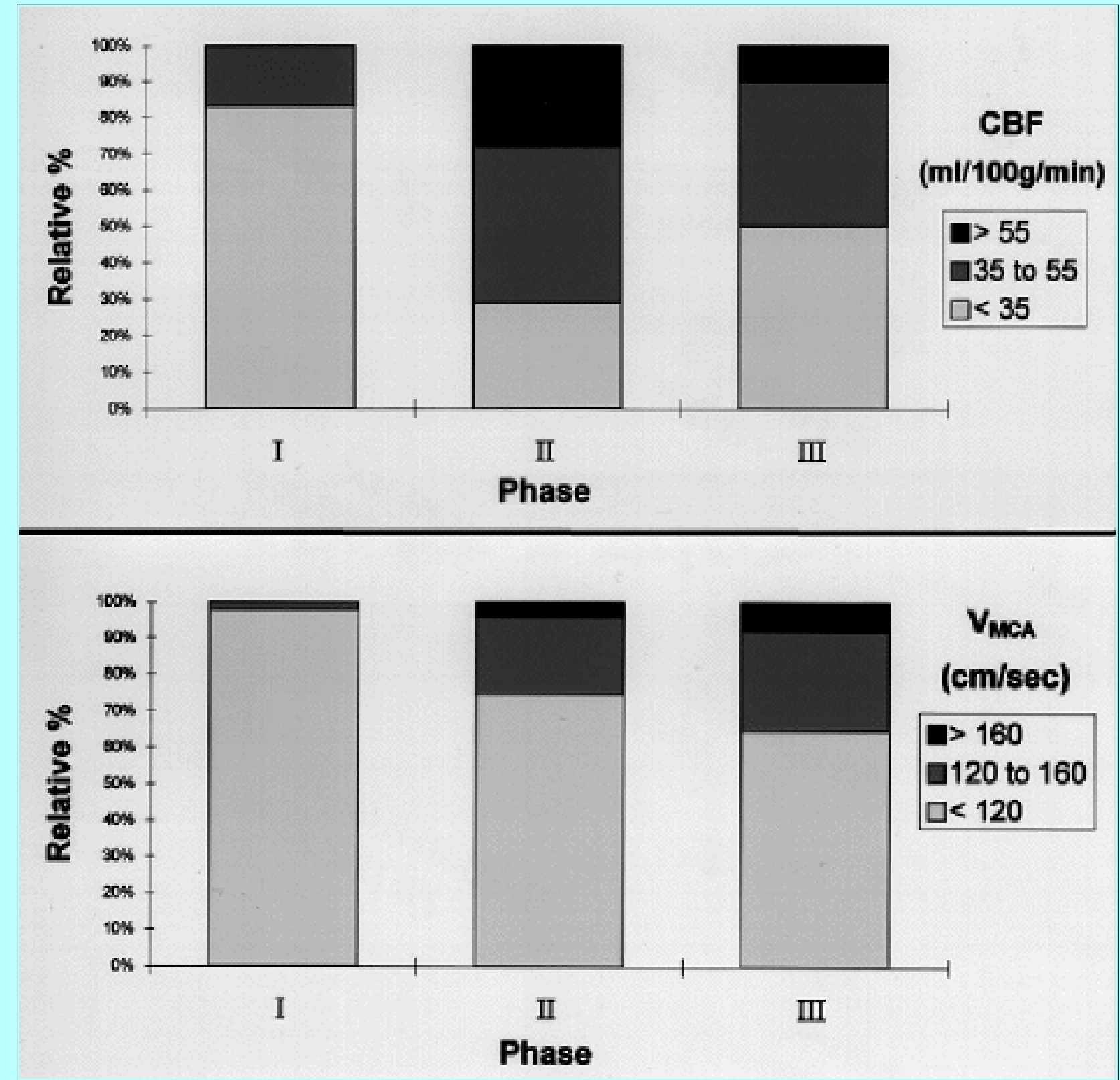

Fig. 2. Bar graphs showing distribution of CBF values and MCA velocity values observed after severe head trauma. Upper: This figure presents, for each phase, the relative percentage of individual CBF studies in which CBF values were within designated ranges (hypoperfusion < $35 \mathrm{ml} / 100 \mathrm{~g} /$ minute; normal-to-relative hyperemia 35-55 ml/100 g/minute; or absolute hyperemia $>55 \mathrm{ml} / 100 \mathrm{~g} /$ minute. Lower: This figure presents, for each phase, the relative percentage of individual TCD studies in which $\mathrm{V}_{\text {MCA }}$ values were within the designated ranges (no vasospasm $<120 \mathrm{~cm} /$ second; mild-to-moderate vasospasm 120-160 $\mathrm{cm} / \mathrm{second}$; or severe vasospasm $>160 \mathrm{~cm} /$ second).

\section{RESULTS}

\section{Patterns of CBF and Blood Flow Velocity After Head Injury}

The daily mean values ( \pm SEM) of the key parameters derived from CBF and TCD studies are illustrated graphically in Fig. 1 upper and center. For comparison, previous studies have described the normal adult mean $\mathrm{V}_{\mathrm{MCA}}$ as 34 to $86 \mathrm{~cm} / \mathrm{second}$ and the mean $\mathrm{V}_{\mathrm{EC}-\mathrm{ICA}}$ as 28 to $46 \mathrm{~cm} / \mathrm{second}$.[2] Earlier studies 
using the intravenous ${ }^{133} \mathrm{Xe}$ method for determining $\mathrm{CBF}$ have shown normal $\mathrm{CBF}_{15}$ to be $44.1 \pm 5.6$ $\mathrm{ml} / 100 \mathrm{~g} / \mathrm{minute}$ at a $\mathrm{PCO}_{2}$ of $34 \mathrm{~mm} \mathrm{Hg.[43]}$

\begin{tabular}{|c|c|c|c|}
\hline \multicolumn{4}{|c|}{$\begin{array}{r}\text { TABLE } 1 \\
\text { CEREERALL BLOOD FLOW AND PHYS }\end{array}$} \\
\hline \multirow[b]{2}{*}{ Parameter } & \multicolumn{3}{|c|}{ Phases } \\
\hline & I(21 patients) & II [54 patients] & III ( 60 patients) \\
\hline 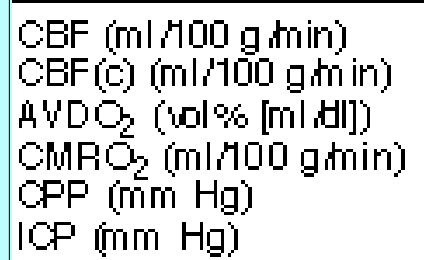 & $\begin{aligned} 33.6 & \pm 1.6 \\
32.3 & \pm 2.0 \\
5.4 & \pm 0.5 \\
1.77 & \pm 0.18 \\
76.7 & \pm 4.0 \\
10.7 & \pm 1.5\end{aligned}$ & $\begin{aligned} 44.0 & \pm 2.4 \dagger \\
46.8 & \pm 3.0 \dagger \\
3.8 & \pm 0.1 \dagger \\
1.49 & \pm 0.82 \\
79.0 & \pm 2.3 \\
15.6 & \pm 1.5\end{aligned}$ & $\begin{aligned} 36.9 & \pm 3.1 \dagger \neq \\
35.7 & \pm 3.8 \dagger \neq \\
3.9 & \pm 0.1 \\
1.46 & \pm 0.56 \\
82.2 & \pm 4.0 \\
16.0 & \pm 2.9\end{aligned}$ \\
\hline $\begin{array}{l}\text { "Values represent th } \\
\mathrm{PCO}_{2} \text { of } 34 \mathrm{~mm} \mathrm{Hg} \text {. } \\
\dagger \mathrm{p}<0.01 \text { iersus } \mathrm{pr} \\
\pm \mathrm{p}<0.01 \text { iersus }\end{array}$ & SEM. At & & \\
\hline
\end{tabular}

Simultaneous analysis of the daily mean CBF and MCA velocities reveals that the data conform to the pattern defined in our pilot study (Tables 1-3).[69] There are three distinct posttraumatic hemodynamic phases (Table 3). Phase I (the first 24 hours after injury) is referred to as the hypoperfusion phase and is defined by a low $\mathrm{CBF}$ (mean corrected $\mathrm{CBF}_{15} 32.3 \pm 2 \mathrm{ml} / 100 \mathrm{~g} /$ minute), a normal $\mathrm{V}_{\text {MCA }}$ (mean $\mathrm{V}_{\mathrm{MCA}}$ $56.7 \pm 2.9 \mathrm{~cm} / \mathrm{second}$ ), and a normal HI (mean HI $1.67 \pm 0.11$ ). In Phase II (hyperemia phase, Days 1-3) the $\mathrm{CBF}$ is transiently increased (mean corrected $\mathrm{CBF}_{15} 46.8 \pm 3 \mathrm{ml} / 100 \mathrm{~g} /$ minute), the $\mathrm{V}_{\mathrm{MCA}}$ is rapidly rising (mean $\mathrm{V}_{\mathrm{MCA}} 86 \pm 3.7 \mathrm{~cm} / \mathrm{second}$ ), and the $\mathrm{HI}$ is normal (mean $\mathrm{HI} 2.40 \pm 0.1$ ). The third phase (vasospasm phase, Days 4-15) is characterized by a variable but gradually declining CBF (mean corrected $\mathrm{CBF}_{15} 35.7 \pm 3.8 \mathrm{ml} / 100 \mathrm{~g} /$ minute; lowest corrected $\mathrm{CBF}_{15} 32.7 \pm 6 \mathrm{ml} / 100 \mathrm{~g} / \mathrm{minute}$ on Day 8). The $\mathrm{V}_{\mathrm{MCA}}$ increases significantly in this phase (mean $\mathrm{V}_{\mathrm{MCA}} 96.7 \pm 6.3 \mathrm{~cm} / \mathrm{second}$; highest $\mathrm{V}_{\mathrm{MCA}}$ $111 \pm 10.2 \mathrm{~cm} /$ second on Day 11), and the HI rises significantly (mean HI $2.87 \pm 0.22$ ).

\begin{tabular}{|c|c|c|c|}
\hline \multicolumn{4}{|c|}{$\begin{array}{c}\text { TABLE } 2 \\
\text { TRANSCRANIAL DOPPLER PARAMETERS FOR EACH PHASE* }\end{array}$} \\
\hline \multirow[b]{2}{*}{ Parameter } & \multicolumn{3}{|c|}{ Phases } \\
\hline & I (42 patients) & II (101 patients) & III [99 patients] \\
\hline 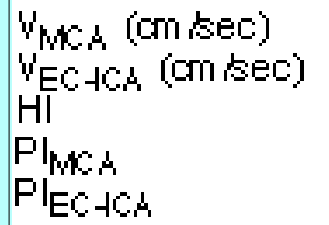 & $\begin{array}{l}56.7 \pm 2.9 \\
34.2 \pm 1.9 \\
1.67 \pm 0.11 \\
1.13 \pm 0.1 \\
1.27 \pm 0.07\end{array}$ & $\begin{array}{l}86.0 \pm 3.7 \dagger \\
36.8 \pm 1.2 \\
2.40 \pm 0.1 \dagger \\
0.98 \pm 0.06 \dagger \\
1.05 \pm 0.04 \dagger\end{array}$ & $\begin{array}{l}96.7 \pm 6.3 \dagger \ddagger \\
34.5 \pm 2.1 \dagger \\
2.87 \pm 0.22 \dagger \ddagger \\
0.94 \pm 0.06 \ddagger \\
1.11 \pm 0.06 \dagger \ddagger\end{array}$ \\
\hline $\begin{array}{l}\text { Talues represe } \\
\dagger p<0.01 \text { versu } \\
\ddagger p<0.01 \text { versu }\end{array}$ & $\begin{array}{l}\text { mean } \pm \text { SEM } \\
\text { vious phase. } \\
\text { ase I. }\end{array}$ & & \\
\hline
\end{tabular}

The phase of hypoperfusion (Phase I) was significantly distinguished from that of hyperemia (Phase II) in terms of lower CBF ( $p<0.01$ ); the vasospasm phase (Phase III) was significantly distinguished from both Phase II (based on a lower CBF, $\mathrm{p}<0.01$ ) and Phase I (based on a higher $\mathrm{V}_{\mathrm{MCA}}[\mathrm{p}<0.01]$ and HI $[\mathrm{p}<0.01])$. 


\begin{tabular}{|c|c|c|c|}
\hline SUMMAR' & RAL HEMOD & ASES FOLLOW & HEAD INWURY \\
\hline \multirow[b]{2}{*}{ Parameter } & \multicolumn{3}{|c|}{ Phases } \\
\hline & I (Day 0) & II [Days 1-3] & III (Days 4-15) \\
\hline \multirow{5}{*}{$\begin{array}{l}\text { CBF } \\
\text { WMCA } \\
\text { HI } \\
\text { A }{ }^{2} O_{2} \\
\text { CMRO2 }\end{array}$} & low & normal high & low \\
\hline & normal & in term ediate & high \\
\hline & normal & in term ediate & high \\
\hline & normal ihigh & $10 w$ & low \\
\hline & low & low & low \\
\hline
\end{tabular}

\section{Individual Variations in CBF and Blood Flow Velocity}

Figure 2 demonstrates the distributions of $C B F$ values and $V_{M C A}$ values that were observed during each phase. Although these graphs demonstrate the prevalence of hypoperfusion during Phase I, hyperemia during Phase II, and vasospasm during Phase III, it is clear that not every patient followed the typical temporal pattern illustrated in Fig. 1 upper and center. A review of Fig. 2 demonstrates that approximately $50 \%$ of the patients have CBF profiles that are consistent with the "average" pattern. Approximately $40 \%$ of the patients appear to demonstrate a $\mathrm{V}_{\text {MCA }}$ pattern consistent with both. Furthermore, when the subset of patients who have CBF and TCD measurements in each of the three phases are reviewed case by case, patterns similar to those presented in Fig. 1 upper and center prevail and are seen in 40 to $50 \%$ of the patients. Although a number of variations on the average temporal profile are possible, two other patterns are seen in a significant subgroup of patients: 1) persistence of hypoperfusion through all three phases and 2) early onset and persistence of hyperemia through Phases II and III (each seen in just under $15 \%$ of the patients).

\section{Measurements of $\mathrm{AVDO} \mathrm{O}_{2}$ After Head Injury}

The $\mathrm{AVDO}_{2}$ in normal subjects at a $\mathrm{PaCO}_{2}$ of $34 \mathrm{~mm} \mathrm{Hg}$ has been reported to be 5 to 9.8 vol\%.[43] Jugular venous bulb sampling in our patients indicates that, in Phase I, there is a relatively normal $\mathrm{AVDO}_{2}$ (mean $\mathrm{PaCO}_{2}$-corrected $\mathrm{AVDO}_{2} 5.4 \pm 0.5$ vol\%) (Table 1). However, in Phase II there is a dramatic, significant $\left(\mathrm{p}<0.01\right.$ ) decrease in $\mathrm{AVDO}_{2}$ (mean corrected $\mathrm{AVDO}_{2} 3.8 \pm 0.1$ vol\%) to well below normal values. The $\mathrm{AVDO}_{2}$ measured during Phase III was only slightly higher than that for Phase II. The $\mathrm{CMRO}_{2}$ was relatively stable through all three phases at a level approximately $50 \%$ below normal (normal $\mathrm{CMRO}_{2} 3.3 \pm 0.4 \mathrm{ml} / 100 \mathrm{~g} /$ minute) (Table 1).[43]

\section{Middle Cerebral Artery and Extracranial ICA PIs After Head Injury}

The PIs of the MCA and extracranial ICA wave forms (as measured by TCD ultrasonography) also show distinct phase-specific changes. Normal values for the MCA PI ( $\left.\mathrm{PI}_{\mathrm{MCA}}\right)$ have been reported as 0.58 to 0.84 with the extracranial ICA PI $\left(\mathrm{PI}_{\mathrm{EC}-\mathrm{ICA}}\right)$ slightly higher due to higher resistance in the ophthalmic distribution.[2] The PI of the MCA is similar to that of the extracranial ICA during Phases I and II: both are higher than normal during Phase $\mathrm{I}\left(\mathrm{PI}_{\mathrm{MCA}} 1.13 \pm 0.1 ; \mathrm{PI}_{\mathrm{EC}-\mathrm{ICA}} 1.27 \pm 0.07\right)$ and both fall toward normal values during Phase II ( $\mathrm{PI}_{\mathrm{MCA}} 0.98 \pm 0.06$; $\left.\mathrm{PI}_{\mathrm{EC}-\mathrm{ICA}} 1.05 \pm 0.04\right)$ (Table 2$)$. The PIs in these two vessels, however, diverge during Phase III $\left(\mathrm{PI}_{\mathrm{MCA}} 0.94 \pm 0.06\right.$; $\left.\mathrm{PI}_{\mathrm{EC}-\mathrm{ICA}} 1.11 \pm 0.06\right)$ (Table 2$)$. 


\section{Intracranial Pressure and CPP After Head Injury}

The mean ICP values were above $10 \mathrm{~mm} \mathrm{Hg}$ all 15 days after injury (reaching a maximum on Day 10 of $18.2 \pm 2.1 \mathrm{~mm} \mathrm{Hg}$ ) (Table 1). Although individual ICP recordings were often more than $20 \mathrm{~mm} \mathrm{Hg}$, the daily mean for the group as a whole never exceeded $20 \mathrm{~mm} \mathrm{Hg}$ due to the aggressive ICP management that was a part of the standard treatment protocol. The mean daily CPP values for the group remained stable between 70 and $90 \mathrm{~mm} \mathrm{Hg}$ throughout the entire course (Fig. 1 lower).

\section{Ischemia During the Three Phases}

None of the 21 patients in whom CBF measurements were obtained during Phase I demonstrated severe hypoperfusion $\left(\mathrm{CBF}_{15}\right.$, or $\mathrm{PCO}_{2}$-corrected $\mathrm{CBF}_{15} \pm 20 \mathrm{ml} / 100 \mathrm{~g} /$ minute $)$. One patient had an $\left(\mathrm{CO}_{2}\right.$ -uncorrected) $\mathrm{AVDO}_{2}$ measurement of 10.3 vol\% when his $\mathrm{PCO}_{2}$ was $22 \mathrm{~mm} \mathrm{Hg}$ (no CBF measurement was made at the time). No other patient had a $\left(\mathrm{CO}_{2}\right.$-uncorrected) $\mathrm{AVDO}_{2}$ above 7 vol\% during Phase I. It should be noted that only three of $21 \mathrm{CBF}$ studies and no $\mathrm{AVDO}_{2}$ measurements in this report were made before 12 hours postinjury. During Phase II, three of $100\left(\mathrm{CO}_{2}\right.$-uncorrected $) \mathrm{CBF}$ measurements showed the $\mathrm{CBF}_{15}$ to be less than $20 \mathrm{ml} / 100 \mathrm{~g} /$ minute. No $\left(\mathrm{CO}_{2}\right.$-uncorrected) $\mathrm{AVDO}_{2}$ measurements were above 8 vol\% and only three of 95 values were over 7 vol\% $\left(\mathrm{PCO}_{2} 3 / 430 \mathrm{~mm} \mathrm{Hg}\right.$ in these three cases). During Phase III, 28 of $182\left(\mathrm{CO}_{2}\right.$-uncorrected) $\mathrm{CBF}$ measurements demonstrated the $\mathrm{CBF}_{15}$ to be less than $20 \mathrm{ml} / 100 \mathrm{~g} /$ minute. No $\left(\mathrm{CO}_{2}\right.$-uncorrected) $\mathrm{AVDO}_{2}$ values were over $8 \mathrm{vol} \%$ and only four of 269 measurements were above 7 vol\% (all with $\mathrm{PCO}_{2} 3 / 431 \mathrm{~mm} \mathrm{Hg}$ ).

\section{Hyperemia During the Three Phases}

The pattern defined by the daily CBF values showed a higher mean flow during postinjury Days 1 to 3, but there was considerable heterogeneity in the CBF measurements for postinjury Phases II and III (Figs. 1 upper and 2 upper). Phase I was the most homogeneous, with only $16.7 \%$ of the CBF values falling in the hyperemic range. Phase II had the widest variety of values, with $\mathrm{CBF}_{15}$ values ranging from 13 to 99 $\mathrm{ml} / 100 \mathrm{~g} /$ minute. Twenty-seven percent of the Phase II CBF values were in the range of absolute hyperemia (>=55 ml/100 g/minute). Ten percent of the Phase II CBF values were greater than $70 \mathrm{ml} / 100$ $\mathrm{g} /$ minute, which represented $75 \%$ of all of the very high CBF values recorded in the first 2 weeks. Overall, $38.8 \%$ of patients studied during this phase had at least one Day 1 to $3 \mathrm{CBF}$ measurement above $55 \mathrm{ml} / 100 \mathrm{~g} / \mathrm{minute}$. Postinjury Days 4 and 5 represented a transition from Phase II to Phase III: almost all of the Phase III CBF values above $55 \mathrm{ml} / 100 \mathrm{~g} /$ minute were seen during these 2 days. Beyond Day 5 , most of the CBF values were low.

\section{Vasospasm During the Three Phases}

Vasospasm of the MCA was defined as ipsilateral $\mathrm{V}_{\text {MCA }}$ above $120 \mathrm{~cm} / \mathrm{second}$ with an $\mathrm{HI}$ above 3 . Using this criterion, we found that $50.4 \%$ of patients had at least one TCD study consistent with vasospasm during their hospital course. Overall, $37.6 \%$ of the patients were classified as having mild-to-moderate spasm ( $\mathrm{V}_{\mathrm{MCA}} 120-160 \mathrm{~cm} /$ second and $\left.\mathrm{HI} 3-4\right)$, and $12.8 \%$ were classified as having severe $\operatorname{spasm}\left(\mathrm{V}_{\mathrm{MCA}}>=160 \mathrm{~cm} /\right.$ second and $\left.\mathrm{HI}>=4\right)$.

The time course of the spasm is apparent (Figs. 1 upper and 2 lower). Only one patient had a $\mathrm{V}_{\mathrm{MCA}}$ greater than $120 \mathrm{~cm} / \mathrm{second}$ during the first 24 hours after injury (Fig. 2 lower). Although some patients 
developed mild-to-moderate spasm during Phase II, almost all of the severe cases of spasm were seen during Phase III (Fig. 2 lower). However, it is important to note that during Phase III there were fewer TCD studies demonstrating evidence of spasm than CBF studies showing low blood flow (that is, many low CBF measurements during Phase III were not associated with vasospasm).

\section{DISCUSSION}

This prospective study has confirmed the findings of a pilot study that reported time-dependent changes in $\mathrm{CBF}, \mathrm{V}_{\mathrm{MCA}}$, and $\mathrm{HI}$, which, considered together, defined three discrete hemodynamic phases (Table 3).[69]

\section{Phase I: Hypoperfusion (Day 0)}

During the first 24 hours following craniocerebral trauma, the uncorrected mean CBF was low (33.6 \pm $1.6 \mathrm{ml} / 100 \mathrm{~g} /$ minute). Even when the CBF in these patients, who generally were hypocapnic because of hyperventilation, was corrected to a $\mathrm{PaCO}_{2}$ of $34 \mathrm{~mm} \mathrm{Hg}$, the adjusted $\mathrm{CBF}$ was substantially lower than normal (normal $44.1 \pm 5.6 \mathrm{ml} / 100 \mathrm{~g} /$ minute at a $\mathrm{PCO}_{2}$ of $34 \mathrm{~mm} \mathrm{Hg}$ ).[43] Also using the ${ }^{133} \mathrm{Xe}$ clearance technique, Fieschi, et al.,[17] Overgaard and Tweed,[47] and others[9,13,15,26,44] have demonstrated that CBF is substantially below normal in the early hours after head injury. The quantitative global CBF values obtained by these investigators are remarkably similar to our results. Even using a different CBF-measuring technique (stable Xe-CT scanning), Marion and colleagues[34] and Bouma and associates[7,10] found levels of global CBF similar to those in the present study. Experimental studies in animal head-trauma models have also demonstrated an acute decrease in CBF to approximately $50 \%$ of baseline.[24,40,48,59,65,68]

Extremely low, "ischemic" levels of CBF have been measured very early after the onset of injury. In a small number of ${ }^{133} \mathrm{Xe}-\mathrm{CBF}$ studies conducted within 6 hours of injury, Bouma, et al.,[9] found the mean flow to be $22.5 \pm 5.2 \mathrm{ml} / 100 \mathrm{~g} /$ minute, with a third of their patients demonstrating ischemic levels (CBF $<18 \mathrm{ml} / 100 \mathrm{~g} /$ minute). Using the Xe-CT technique, these investigators found very early evidence of either global or regional ischemia in approximately $30 \%$ of the patients and observed that these patients had a substantially worse outcome than patients without evidence of ischemia.[10] We did not observe such low levels of CBF in our patients, primarily because the first evaluation was not performed within 6 hours after patient admission.

Our studies of cerebral oxygen metabolism rarely demonstrated the very high $\mathrm{AVDO}_{2}$ levels that are the hallmark of ischemia. This is consistent with previously published results.[9,26,43,49,51] However, very early after injury when $\mathrm{CBF}$ is at its lowest, some cases display an $\mathrm{AVDO}_{2}$ in the ischemic range.[9] These findings suggest that hyperacute posttraumatic cerebral ischemia may occur before measurements can generally be made, but indicate that the low global CBF seen in the first 24 hours is often matched to low $\mathrm{CMRO}_{2}$ (Table 1).

Blood flow velocity in the extracranial segment of the ICA and in the proximal MCA was in the normal or low-to-normal range during the first 24 hours after the onset of injury. The mean HI during the 1st postinjury day was also normal. Therefore, the results of the present study and those of other reported studies of posttraumatic blood flow velocity measurements refute the notion that a low CBF during the first 24 hours after head injury is due to acute vasospasm of the large basal cerebral arteries.[9,36,37,60] 
What might be the cause of the low CBF seen in the first hours after head injury? The data presented in this report, supported by findings of other investigators, demonstrate that the impairment in CBF cannot be attributed to abnormally low CPP, excessive hyperventilation, or vasospasm. $[9,36,37,44]$ The cerebral microcirculation appears to be the location of increased resistance causing early posttraumatic hypoperfusion. Our TCD studies have demonstrated that the PIs (which are increased by high distal vascular resistance) are elevated in both the extracranial ICA and the MCA. This strongly suggests the presence of high distal (microcirculatory) vascular resistance. The finding of subnormal cerebral blood volume during the first 24 hours postinjury also suggests narrowing of the microcirculation.[7]

The cause of the narrowing in the microcirculation may be multifactorial. Extrinsic microvascular compression by damaged and edematous astrocytic processes has been observed in experimental models of head trauma and in specimens of damaged human brain tissue removed at surgery.[23,53] Active muscular constriction of the resistance arterioles might be caused by trauma-induced release of vasoactive substances such as calcium, catecholamines, prostaglandins, or neuropeptides (endothelin, neuropeptide Y).[18,22,25,38,55,61,62,66] Hemoglobin, released as a consequence of posttraumatic subarachnoid hemorrhage, has also been demonstrated to be a vasoconstrictor.[61] Additionally, intravascular thrombosis may play a role in trauma-induced microcirculatory obstruction.[23] Given the normal $\mathrm{AVDO}_{2}$ values seen in most cases, posttraumatic hypoperfusion may also often represent appropriate coupling of low CBF to trauma-induced depression of cerebral oxidative metabolism.

\section{Phase II: Hyperemia (Days 1-3)}

The hypoperfusion found during the first 24 hours after head injury is followed by progressively increasing CBF (Fig. 1 upper). In fact, $\mathrm{CBF}$ probably begins rising within hours from the lowest levels immediately after injury. The increase in CBF reaches its maximum between 48 and 72 hours after the injury (postinjury Day 2 in this report). Overgaard and Tweed,[47] Obrist and colleagues,[42] Bouma and associates,[7-9] and others[14,42,44,50] have described a similar time course for posttraumatic hyperemia using the ${ }^{133} \mathrm{Xe}$ clearance technique, the Kety-Schmidt (nitrous oxide saturation) technique, and stable Xe-CT scanning. The finding of increased CBF using these techniques is confirmed by the observation of increasing velocity in the extracranial segment of the ICA (Table 2). The increase in CBF elicits an increase in $\mathrm{V}_{\mathrm{MCA}}$ as well, although in some cases this may be due to the early onset of cerebral arterial spasm (manifested by an increase in the HI).

In many cases the $\mathrm{CBF}$ rose from the subnormal levels of the first phase into a range that would be considered normal for a nontraumatized healthy adult. As stated by Obrist and colleagues,[43] a "normal" CBF can be considered "relative hyperemia" in a comatose trauma patient, in whom normal metabolic coupling would result in a level of CBF considerably below normal. However, almost $40 \%$ of the patients during postinjury Days 1 to 3 demonstrated "absolute hyperemia" (with at least one measurement of $\mathrm{CBF}$ above the upper limit of the "normal" $\mathrm{CBF}$ range).

The hyperemia phase includes postinjury Days 1 through 3 (Hours 24-96). However, some hyperemic CBF measurements were recorded after Day 3 (Fig. 2 upper). The great majority of these few Phase III hyperemic CBF measurements were seen on postinjury Days 4 and 5, indicating that these days represented a transition between Phase II and Phase III.

The clinical significance of hyperemia has not been fully defined. Clinical studies conducted by Bruce, et al.,[11] and Obrist and colleagues[43] suggest that hyperemia is the primary cause of increased ICP. 
Other recent studies, however, have failed to confirm the correlation between elevated CBF and increased ICP.[41,49] The relationship of hyperemia to clinical outcome is also unclear.[26,41,49] Our group has recently completed an analysis of posttraumatic hyperemia and found that hyperemia-associated intracranial hypertension occurs in almost $15 \%$ of patients with moderate-to-severe head injury and is predictive of a poor outcome.[29] Early identification and aggressive treatment of these patients might improve their prognosis. Mild hyperemia without intracranial hypertension, conversely, is linked to favorable outcome in our experience.[29]

The pathophysiological mechanisms that produce posttraumatic hyperemia remain obscure. The data presented in this report indicate that increases in mean arterial pressure or CPP are not responsible for this hemodynamic change. Hyperemia is not induced by an increase in the $\mathrm{CMRO}_{2}$, as demonstrated by the results reported in this paper and in other work. $[9,43]$ In fact, a certain degree of uncoupling of blood flow from oxygen metabolism seems to occur. However, the possibility exists that this hyperemia may occur in response to an increased cerebral metabolic rate of glucose. Posttraumatic hyperglycolysis has been identified in animal models of head trauma by Andersen and Marmarou,[4] Hovda, et al.,[24] and Yoshino and colleagues.[67] Recent positron emission tomography studies of glucose metabolism in patients with head trauma at UCLA have demonstrated global and regional hyperglycolysis in some patients during this time period.[5,6]

The PIs in both the ICA and the MCA fall on postinjury Day 1 (Table 2), providing evidence that the hyperemic phase is associated with a drop in distal cerebrovascular resistance. The generation of vasodilatory metabolites (lactic acid, neuropeptides, and adenosine) has been associated with hyperemia, or "luxury perfusion," following ischemic damage to the brain and may play a role in posttraumatic hyperemia.[13,14,32,64] Alternatively, there may be a delayed onset of mechanically induced vascular smooth-muscle dysfunction (or "vasoparalysis") that results in a decrease in cerebrovascular resistance and an increase in blood flow.[20]

The cause of the termination of the hyperemic phase is not entirely clear. This may result from the clearance of vasodilatory metabolites, recovery of vascular smooth-muscle function, or resolution of posttraumatic hyperglycolysis. However, examination of the MCA PIs indicates that this phase is not necessarily terminated by an increase in distal vascular resistance (Table 2). Our data indicate that the hyperemic phase may resolve, in part, because large artery spasm occurs.

\section{Phase III: Vasospasm (Days 4-14)}

In the third phase, the physiological findings include increasing $\mathrm{V}_{\mathrm{MCA}}$ and an increasing HI (Fig. 1 center and Table 2). These findings are consistent with the onset of vasospasm involving the proximal segment of the MCAs. The increase in the $\mathrm{V}_{\mathrm{MCA}}$ is clearly due to progressive arterial narrowing and not to a flow effect, because CBF falls during this phase.[12,20,36] Furthermore, an increasing PI found in the cervical segment of the ICA indicates an increase in downstream resistance, presumably due to the narrowing of the MCA. Approximately $50 \%$ of our patients developed significant MCA spasm, evidenced by MCA velocities greater than $120 \mathrm{~cm} / \mathrm{second}$ and by a concomitant HI over 3 . Although $\mathrm{AVDO}_{2}$ recordings during Phase III rarely demonstrated the high levels associated with ischemia, focal vasospasm-induced ischemia may have been missed using this global measurement technique.

Until recently, posttraumatic vasospasm had only been defined using angiography.[32,57,63] The largest angiographic study, by Suwanwela and Suwanwela,[57] found that $18.6 \%$ of patients with 
moderate-to-severe head injury had narrowing of at least one intracranial artery. The study of posttraumatic vasospasm using TCD ultrasonography has allowed sequential noninvasive assessment of arterial narrowing following head injury. Weber, et al.,[60] found increased MCA velocities consistent with spasm in $40 \%$ of head-injured patients. Gomez and associates[20] used TCD ultrasonography in combination with angiography to confirm the finding of posttraumatic vasospasm. Our group's 1992 study[36] demonstrated TCD and CBF evidence of vasospasm in 27\% of head-injured patients (with angiographic confirmation in three patients with severe spasm). This study demonstrated that MCA vasospasm began at approximately postinjury Day 4 and persisted for as long as 3 weeks after head injury. The degree of the spasm demonstrated in these studies has, in some cases, been as severe as that seen in patients with aneurysmal subarachnoid hemorrhage who develop delayed ischemic deficit. A more recent report published by our center, based on a larger group of patients, suggests that posttraumatic spasm is associated with poor clinical outcome.[37]

However, less than $15 \%$ of patients have TCD findings that suggest severe vasospasm, whereas almost $50 \%$ of CBF measurements during Phase III show low flow. It is probable that low CBF during this phase is often coupled to persistent posttraumatic hypometabolism. The measurements of cerebral oxygen metabolism obtained during this time demonstrate that $\mathrm{CMRO}_{2}$ remains approximately $50 \%$ of normal. Recent positron emission tomography studies (using [ $\left.{ }^{18} \mathrm{~F}\right]$ fluorodeoxyglucose) in head-injured patients have shown that the cerebral metabolic rate of glucose is also suppressed beyond the first 4 or 5 days after injury.[5,6] Therefore, this phase includes vasospasm in some cases, and low CBF coupled to low cerebral metabolism in others.

\section{Resolution of Posttraumatic Hemodynamic Changes}

This research study does not address the late resolution of trauma-induced hemodynamic disturbances because of the paucity of CBF or TCD evaluations performed beyond 2 weeks after injury (most patients have been discharged or transferred by this time). The few cases that had later measurements (reported in our pilot study) demonstrated gradual recoveries toward normal on the part of CBF and MCA velocities beginning 2 to 3 weeks after injury.[69] A previously published study of posttraumatic vasospasm also showed resolution of even the most severe cases after 3 to 4 weeks.[36] It seems that the fourth phase of the posttraumatic cerebral hemodynamic pattern should be termed the "recovery phase," beginning 2 to 3 weeks after onset and probably lasting weeks to months.

\section{Shortcomings, Flaws, and Sources of Bias}

Because of the logistical problems involved in performing TCD and CBF evaluations during the 1st clinically busy hours after admission to the emergency department, we have obtained few studies within 12 hours of injury. The earliest cerebral hemodynamic and metabolic alterations must be inferred from the few other clinical studies that have focused on the earliest hours and from laboratory investigations. The true prevalence of hyperacute ischemia (within the first 1 or 2 hours) in head-injured patients remains to be determined.

The CBF and metabolic measurements reported in this paper represent global alterations. Focal disturbances in flow or metabolism caused by discrete traumatic lesions (hematomas or contusions) or related to isolated vascular pathology (focal spasm or dissection) will be "blurred" or may be missed entirely. Furthermore, subcortical, cerebellar, and brainstem CBF and metabolism are not specifically evaluated using the techniques discussed in this work. For regional flow and metabolism measurements 
one must refer to autoradiographic studies conducted in experimental models of head trauma or to the few clinical studies performed with tomographic techniques.[7,10,34] Positron emission tomography (using ${ }^{15} \mathrm{O}$-containing tracers for $\mathrm{CBF}$ and oxygen metabolism measurements and [18F]fluorodeoxyglucose for glucose metabolism measurements) is the most promising, although logistically difficult, technique for defining pathophysiology on a regional scale. We have begun such studies and plan to extend these over the next several years.[5,6]

One of the inherent flaws in this project is the inability to study patients who have been transferred to other medical facilities or rehabilitation units or discharged from the hospital. Therefore, it is likely that the data in later phases are skewed toward patients with more severe injuries and more complicated hospital stays.

It is important to recognize that not every patient experienced the typical hemodynamic alterations during each postinjury phase (Fig. 2). Studies in progress will address CBF changes and vasospasm in clinically important subgroups (stratified by age, CT findings, presence or absence of intracranial hypertension, outcome, and so forth). In a fashion similar to that used by Robertson and colleagues,[49] future studies must address the importance of variations on the "average" temporal profile of hemodynamic disturbances.

\section{Potential Therapeutic Implications}

In Phase I, hypoperfusion may be a critical secondary insult to the traumatized brain, with ischemia and infarction compounding the initial mechanical injury. Phase I, therefore, requires rigorous maintenance of normal CPP, normal oxygenation, and a normal hematocrit level. In general, profound hyperventilation should be avoided because, as Sheinberg, et al.,[54] have demonstrated, hypocarbia may lead to more profound hypoperfusion and a concomitant poor outcome. Potential strategies for future therapy for the low blood flow of Phase I include induced arterial hypertension; pharmacological agents that might reverse the increase in small vessel resistance that appears to be present; or therapies (drugs or hypothermia) that protect neurons from ischemia-induced biochemical events.

Recent work performed at our center and others has indicated that the hyperemia of Phase II may be associated with harmful intracranial hypertension.[11,29,43] Because this phase is not associated with MCA spasm or usually with a high $\mathrm{AVDO}_{2}$ indicating inadequate oxygen delivery, hyperventilation should be safe during Phase II. During this phase, the prominence of hyperemia suggests that "CPP therapy" using pressor-induced arterial hypertension should be used cautiously and should, perhaps, be guided by CBF measurements.[52]

Phase III appears to be a more important phase than generally appreciated. It is reasonable to propose that the vasospasm that occurs in some patients in Phase III should be monitored using TCD ultrasonography and treated in the same way as vasospasm that follows aneurysmal subarachnoid hemorrhage--with volume expansion and induced arterial hypertension.[28,45] Treatment with nimodipine (or yet-to-be developed drugs) may reduce the ischemic neurological damage that may occur during this phase. For particularly severe symptomatic cases, balloon angioplasty may be considered.[16]

\section{Acknowledgments}

The authors wish to thank Walter Obrist, Ph.D., David Stump, Ph.D., and David Beyda, M.D., for their advice and assistance with the CBF measurement technique. We would also like to acknowledge 
Kathleen Thomas-Lukes, Brenda Rinsky, and Oscar Barcenas for performing the TCD studies presented in this report, and Eula Mckinney and Karen Einstein for secretarial assistance.

\section{References}

1. Aaslid R, Markwalder TH, Nornes H: Noninvasive transcranial Doppler ultrasound recording of flow velocity in basal cerebral arteries. J Neurosurg 57:769-774, 1982

2. Adams RJ, Nichols FT, Hess DC: Normal values and physiological variables, in Newell DW, Aaslid R (eds): Transcranial Doppler. New York: Raven Press, 1992, pp 41-48

3. Agnoli A, Prencipe M, Priori AM, et al: Measurements of the rCBF by intravenous injection of ${ }^{133} \mathrm{Xe}$. A comparative study with the intra-arterial injection method, in Brock M, Fieschi C, Ingvar DH, et al (eds): Cerebral Blood Flow. Clinical and Experimental Results. Berlin: Springer-Verlag, 1969, pp $31-34$

4. Andersen BJ, Marmarou A: Post-traumatic selective stimulation of glycolysis. Brain Res 585:184-189, 1992

5. Bergsneider M, Hovda DA, Shalmon E, et al: Cerebral hyperglycolysis following severe traumatic brain injury in humans: a positron emission tomography study. J Neurosurg 86:241-251, 1997

6. Bergsneider M, Kelly DF, Shalmon E, et al: Dynamic changes in cerebral glucose metabolism following severe head injury as measured by positron emission tomography. J Cereb Blood Flow Metab 15 (Suppl 1):S26, 1995 (Abstract)

7. Bouma GJ, Muizelaar JP: Cerebral blood flow, cerebral blood volume, and cerebrovascular reactivity after severe head injury. J Neurotrauma 9 (Suppl 1):S333-S348, 1992

8. Bouma GJ, Muizelaar JP: Evaluation of regional cerebral blood flow in acute head injury by stable xenon-enhanced computerized tomography. Acta Neurochir Suppl 59:34-40, 1993

9. Bouma GJ, Muizelaar JP, Choi SC, et al: Cerebral circulation and metabolism after severe traumatic brain injury: the elusive role of ischemia. J Neurosurg 75:685-693, 1991

10. Bouma GJ, Muizelaar JP, Stringer WA, et al: Ultra-early evaluation of regional cerebral blood flow in severely head-injured patients using xenon-enhanced computerized tomography. J Neurosurg 77:360-368, 1992

11. Bruce DA, Langfitt TW, Miller JD, et al: Regional cerebral blood flow, intracranial pressure, and brain metabolism in comatose patients. J Neurosurg 38:131-144, 1973

12. Chan KH, Dearden NM, Miller JD: The significance of posttraumatic increase in cerebral blood flow velocity: a transcranial Doppler ultrasound study. Neurosurgery 30:697-700, 1992

13. De Salles AAF, Muizelaar JP, Young HF: Hyperglycemia, cerebrospinal fluid lactic acidosis, and cerebral blood flow in severely head-injured patients. Neurosurgery 21:45-50, 1987

14. Enevoldsen EM, Cold G, Jensen FT, et al: Dynamic changes in regional CBF, intraventricular pressure, CSF pH and lactate levels during the acute phase of head injury. J Neurosurg 44:191-214, 
15. Enevoldsen EM, Jensen FT: Compartmental analysis of regional cerebral blood flow in patients with acute severe head injuries. J Neurosurg 47:699-712, 1977

16. Eskridge JM, Newell DW, Pendleton GA: Transluminal angioplasty for treatment of vasospasm. Neurosurg Clin North Am 1:387-399, 1990

17. Fieschi C, Battistini N, Beduschi A, et al: Regional cerebral blood flow and intraventricular pressure in acute head injuries. J Neurol Neurosurg Psychiatry 37:1378-1388, 1974

18. Fineman I, Hovda DA, Smith M, et al: Concussive brain injury is associated with a prolonged accumulation of calcium: a ${ }^{45} \mathrm{Ca}$ autoradiographic study. Brain Res 624:94-102, 1993

19. Gobiet W, Grote W, Bock WJ: The relation between intracranial pressure, mean arterial pressure and cerebral blood flow in patients with severe head injury. Acta Neurochir 32:13-24, 1975

20. Gomez CR, Backer RJ, Bucholz RD: Transcranial Doppler ultrasound following closed head injury: vasospasm or vasoparalysis? Surg Neurol 35:30-35, 1991

21. Graham DI, Adams JH, Doyle D: Ischaemic brain damage in fatal non-missile head injuries. J Neurol Sci 39:213-234, 1978

22. Hayes RL, Jenkins LW, Lyeth BG: Neurotransmitter-mediated mechanisms of traumatic brain injury: acetylcholine and excitatory amino acids. J Neurotrauma 9 (Suppl 1):S173-S187, 1992

23. Hekmatpanah J, Hekmatpanah CR: Microvascular alterations following cerebral contusion in rats. Light, scanning, and electron microscope study. J Neurosurg 62:888-897, 1985

24. Hovda DA, Lee SM, Smith ML, et al: The neurochemical and metabolic cascade following brain injury: moving from animal models to man. J Neurotrauma 12:903-906, 1995

25. Huger F, Patrick G: Effect of concussive head injury on central catecholamine levels and synthesis rates in rat brain regions. J Neurochem 33:89-95, 1979

26. Jaggi JL, Obrist WD, Gennarelli TA, et al: Relationship of early cerebral blood flow and metabolism to outcome in acute head injury. J Neurosurg 72:176-182, 1990

27. Jakobsen M, Enevoldsen E: Retrograde catheterization of the right internal jugular vein for serial measurements of cerebral venous oxygen content. J Cereb Blood Flow Metab 9:717-720, 1989

28. Kassell NF, Peerless SJ, Durward QJ, et al: Treatment of ischemic deficits from vasospasm with intravascular volume expansion and induced arterial hypertension. Neurosurgery 11:337-343, 1982

29. Kelly DF, Kordestani RK, Martin NA, et al: Hyperemia following traumatic brain injury: relationship to intracranial hypertension and outcome. J Neurosurg 85:762-771, 1996

30. Legarth J, Thorup E: Characteristics of Doppler blood-velocity waveforms in a cardiovascular in vitro model. II: The influence of peripheral resistance, perfusion pressure and blood flow. Scand J Clin Lab Invest 49:459-464, 1989

31. Lindegaard KF, Nornes H, Bakke SJ, et al: Cerebral vasospasm diagnosis by means of angiography 
and blood velocity measurements. Acta Neurochir 100:12-24, 1989

32. MacFarlane R, Moskowitz MA, Sakas DE, et al: The role of neuroeffector mechanisms in cerebral hyperperfusion syndromes. J Neurosurg 75:845-855, 1991

33. Macpherson P, Graham DI: Correlation between angiographic findings and the ischaemia of head injury. J Neurol Neurosurg Psychiatry 41:122-127, 1978

34. Marion DW, Darby J, Yonas H: Acute regional cerebral blood flow changes caused by severe head injuries. J Neurosurg 74:407-414, 1991

35. Marmarou A, Ward JD, Young HF, et al: Impact of ICP instability and hypotension on outcome in patients with severe head trauma. J Neurosurg 75 (Suppl):S59-S66, 1991

36. Martin NA, Doberstein C, Alexander M, et al: Posttraumatic cerebral arterial spasm. J Neurotrauma 12:897-901, 1995

37. Martin NA, Doberstein C, Zane C, et al: Posttraumatic cerebral arterial spasm: transcranial Doppler ultrasound, cerebral blood flow, and angiographic findings. J Neurosurg 77:575-583, 1992

38. McIntosh TK, Ferriero D: Changes in neuropeptide Y after experimental traumatic injury in the rat. J Cereb Blood Flow Metab 12:697-702, 1992

39. Miller JD, Dearden NM, Piper IR, et al: Control of intracranial pressure in patients with severe head injury. J Neurotrauma 9 (Suppl 1):S317-S326, 1992

40. Muir JK, Boerschel M, Ellis EF: Continuous monitoring of posttraumatic cerebral blood flow using laser-Doppler flowmetry. J Neurotrauma 9:355-362, 1992

41. Muizelaar JP, Marmarou A, DeSalles AAF, et al: Cerebral blood flow and metabolism in severely head-injured children. Part 1: Relationship with GCS score, outcome, ICP, and PVI. J Neurosurg 71:63-71, 1989

42. Obrist WD, Gennarelli TA, Segawa H, et al: Relation of cerebral blood flow to neurological status and outcome in head-injured patients. J Neurosurg 51:292-300, 1979

43. Obrist WD, Langfitt TW, Jaggi JL, et al: Cerebral blood flow and metabolism in comatose patients with acute head injury. Relationship to intracranial hypertension. J Neurosurg 61:241-253, 1984

44. Obrist WD, Marion DW, Aggarwal S, et al: Time course of cerebral blood flow and metabolism in comatose patients with acute head injury. J Cereb Blood Flow Metab 13:S571, 1993 (Abstract)

45. Origitano TC, Wascher TM, Reichman OH, et al: Sustained increased cerebral blood flow with prophylactic hypertensive hypervolemic hemodilution ("Triple-H" therapy) after subarachnoid hemorrhage. Neurosurgery 27:729-748, 1990

46. Overgaard J, Mosdal C, Tweed WA: Cerebral circulation after head injury. Part 3: Does reduced regional cerebral blood flow determine recovery of brain function after blunt head injury? J Neurosurg 55:63-74, 1981

47. Overgaard J, Tweed WA: Cerebral circulation after head injury. Part 1: Cerebral blood flow and its regulation after closed head injury with emphasis on clinical correlations. J Neurosurg 41:531-541, 
48. Pfenninger EG, Reith A, Breitig D, et al: Early changes of intracranial pressure, perfusion pressure, and blood flow after acute head injury. Part 1: An experimental study of the underlying pathophysiology. J Neurosurg 70:774-779, 1989

49. Robertson CS, Contant CF, Narayan RK, et al: Cerebral blood flow, $\mathrm{AVDO}_{2}$, and neurologic outcome in head-injured patients. J Neurotrauma 9 (Suppl 1):S349-S358, 1992

50. Robertson CS, Grossman RG, Goodman JC, et al: The predictive values of cerebral anaerobic metabolism with cerebral infarction after head injury. J Neurosurg 67:361-368, 1987

51. Robertson CS, Narayan RK, Gokaslan ZL, et al: Cerebral arteriovenous oxygen difference as an estimate of cerebral blood flow in comatose patients. J Neurosurg 70:222-230, 1989

52. Rosner MJ, Rosner SD, Johnson AH: Cerebral perfusion pressure: management protocol and clinical results. J Neurosurg 83:949-962, 1995

53. Schröder ML, Muizelaar JP, Bullock MR, et al: Focal ischemia due to traumatic contusions documented by stable xenon-CT and ultrastructural studies. J Neurosurg 82:966-971, 1995

54. Sheinberg M, Kanter MJ, Robertson CS, et al: Continuous monitoring of jugular venous oxygen saturation in head-injured patients. J Neurosurg 76:212-217, 1992

55. Shohami E, Novikov M, Bass R, et al: Closed head injury triggers early production of TNF alpha and IL-6 by brain tissue. J Cereb Blood Flow Metab 14:615-619, 1994

56. Sorteberg W, Langmoen IA, Lindegaard KF, et al: Side-to-side differences and day-to-day variations of transcranial Doppler parameters in normal subjects. J Ultrasound Med 9:403-409, 1990

57. Suwanwela C, Suwanwela N: Intracranial arterial narrowing and spasm in acute head injury. J Neurosurg 36:314-323, 1972

58. Teasdale G, Jennett B: Assessment of coma and impaired consciousness. A practical scale. Lancet 2:81-84, 1974

59. Velarde F, Fisher DT, Hovda DA, et al: Fluid percussion injury induces prolonged changes in cerebral blood flow. J Neurotrauma 9:402, 1992 (Abstract)

60. Weber M, Grolimund P, Seiler RW: Evaluation of posttraumatic cerebral blood flow velocities by transcranial Doppler ultrasonography. Neurosurgery 27:106-112, 1990

61. White RP: Responses of isolated cerebral arteries to vasoactive agents. Neurosurg Clin North Am 1:401-415, 1990

62. White RP, Hagen AA: Cerebrovascular actions of prostaglandins. Pharmacol Ther 18:313-331, 1982

63. Wilkins RH, Odom GL: Intracranial arterial spasm associated with craniocerebral trauma. J Neurosurg 32:626-633, 1970

64. Winn HR, Rubio R, Berne RM: Brain adenosine concentration during hypoxia in rats. Am J Physiol 
65. Yamakami I, McIntosh TK: Alterations in regional cerebral blood flow following brain injury in the rat. J Cereb Blood Flow Metab 11:655-660, 1991

66. Yanagisawa M, Kurihara H, Kimura S, et al: A novel potent vasoconstrictor peptide produced by vascular endothelial cells. Nature 332:411-415, 1988

67. Yoshino A, Hovda DA, Kawamata T, et al: Dynamic changes in local cerebral glucose utilization following cerebral concussion in rats: evidence of a hyper- and subsequent hypometabolic state. Brain Res 561:106-119, 1991

68. Yuan XQ, Prough DS, Smith TL, et al: The effects of traumatic brain injury on regional cerebral blood flow in rats. J Neurotrauma 5:289-301, 1988

69. Zane CJ, Khanna R, Martin N, et al: Patterns of cerebral blood flow and transcranial Doppler ultrasound velocities following head injury. J Neurosurg 76:399A, 1992 (Abstract)

Manuscript received April 20, 1993.

Accepted in final form January 27, 1997.

This study was supported by Grant No. NS 30308 (UCLA Brain Injury Research Center, D. P. Becker, Principal Investigator; Cerebral Blood Flow Project, N. A. Martin, Project Investigator) from the National Institute for Neurological Disorders and Stroke; and by gifts from the MacDonald Family Foundation, and the Permanent Charities Committee of the Entertainment Industry.

Address reprint requests to: Neil A. Martin, M.D., Division of Neurosurgery, UCLA Medical Center, CHS 74-140, 10833 Le Conte Avenue, Los Angeles, California 90024-7039. email: martin@ neurosurg.medsch.ucla.edu. 